\title{
An Improved M-ary Modulation Scheme Based on Chaotic Dynamics
}

\author{
Hikmat N. Abdullah ${ }^{1}$, Asaad H. Sahar ${ }^{2}$, and Thamir R. Saeed ${ }^{2}$ \\ ${ }^{1}$ Al-Nahrain University, College of Information Engineering, Baghdad, Iraq \\ ${ }^{2}$ Department of Electrical Engineering, University of Technology, Baghdad, Iraq
}

\begin{abstract}
This paper proposes an improved chaos-based M-ary modulation system. It reproduces deterministic chaotic dynamics to create M-ary non-coherent modulation. The proposed modulation system transmits data using autonomous chaotic sequences. It separates the chaotic dynamics through the use of interleavers and realigns them through the use deinterleavers. The simulation results show that the improved scheme overperforms its traditional counterpart. The degree of improvement grows as the $\mathrm{M}$-ary order is increased, with a penalty of increased system complexity.
\end{abstract}

Keywords-chaos, M-ary modulation, non-coherent detection, zigzag map.

\section{Introduction}

The use of chaotic systems in wireless communication is a topic of interest in the field of chaos engineering [1]. Chaotic systems are generated based on the deterministic rule and their signals are characterized by the following properties: they are random-like, non-periodic, bounded, sensitive to initial conditions, difficult in terms of predicting future attitudes based on past perceptions, and wideband [2]. Over the past few decades, most researches have been concentrating on these properties and have made an effort to address the different topics related to chaosbased communications [3]. For instance, the studies performed explored spread-spectrum communication systems, their modulation schemes, chaos-based coding, as well as multiple-access schemes [4]. Chaos-based communication systems are divided into two classes, as they are based on coherent and non-coherent detection [5]. The coherent category requires synchronization of the receiver, while its non-coherent counterpart does not need synchronization between the transmitter and receiver [6]. Several types of research have focused on the non-coherent system which does not use the base signals at the receiver [7], assuming that chaotic sequences should be characterized by unique features themselves. In this regard, non-coherent detection is regarded as a special detection method that relies on the principle of chaos [6]. It is worth noting that optimal and differential chaos shift keying (DCSK) is a part of the non-coherent system [8]. It has been established that the M-ary communication system based on chaos has not been widely researched, and this has been linked to chaos in binary digital communication [9], [10]. There is a gap between traditional coding methods, such as QAM, and the existing method of the chaotic system [11]. As documented by Arai et al. [12], the modulation algorithm relies on separation and rearrangement of chaotic dynamics in order to achieve non-coherent, chaos-based communication. This study proposes an improved M-ary modulation scheme capable of implying security and improving the bit error rate through the use of zigzag mapping and interleaving. The use of a zigzag map demonstrates excellent chaotic behaviors, adopted in truly random number generators (TRNGs) [13].

\section{M-ary Modulation Based on Chaos}

The M-ary modulation transmits $b$ data bits at the same time, as it uses $M=2^{b}$ different chaotic sequences. For instance, one sequence for each $2^{b}$ is used to express a $b$ bit sequence. Current research generates $M$ distinct sequences for separation of chaotic dynamics, as it adopts a chaotic sequence. Additionally, the receiver recovers $b$ data bits, through the reconstruction of the original sequence, in line with the chaotic dynamics from the transmitter. Therefore, the process of separating and rearranging the chaotic dynamics relies on the interleaver (intr $m$ ) and deinterleaver (deint $m$ ), respectively, where $m$ equals $0,1,2,3, \ldots, M-1$. At the transmitter and the receiver, M-ary modulation prepares the $M$ interleavers and deinterleavers. Each interleaver-deinterleaver pair is assigned to one of the data bits through the $M$ symbols. The transmitter helps select an interleaver that aligns the data symbol and helps order the separation of samples in the chaotic sequence. The sequence is fed into all $M$ deinterleavers deinterleavers at the receiver. The original sequence is reconstructed by applying the correct deinterleaver only. Therefore, the receiver recovers $b$ bits of data by taking part in the analysis of the chaotic dynamics for every reconstructed sequence. 


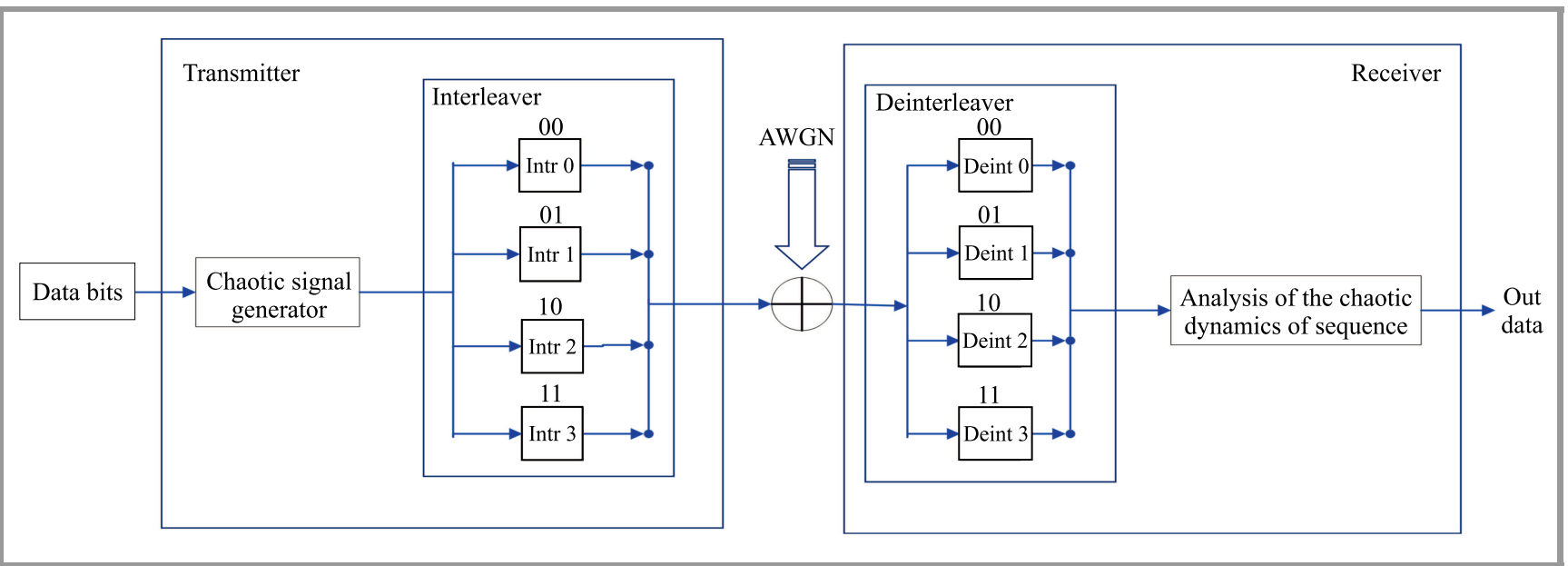

Fig. 1. Improved M-ary modulation structure.

(a)
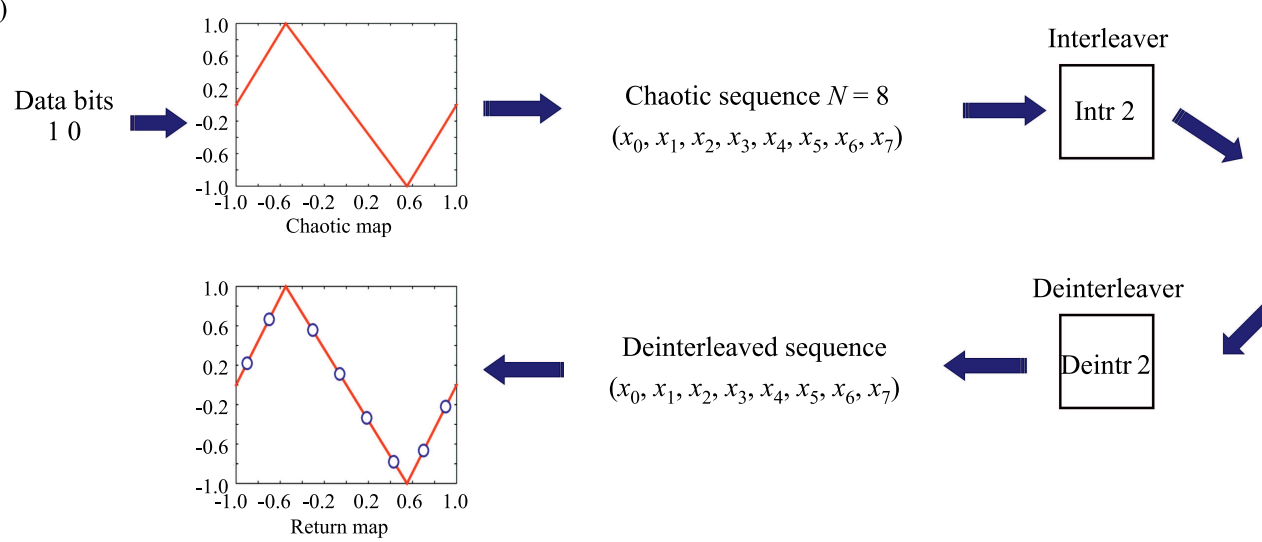

$\left(x_{0}, x_{1}, x_{2}, x_{3}, x_{4}, x_{5}, x_{6}, x_{7}\right)$

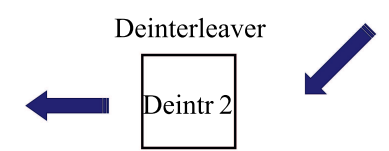

Interleaved sequence

$\left(x_{6}, x_{7}, x_{0}, x_{2}, x_{1}, x_{3}, x_{4}, x_{5}\right)$

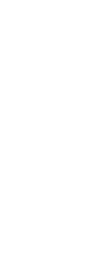

(b)

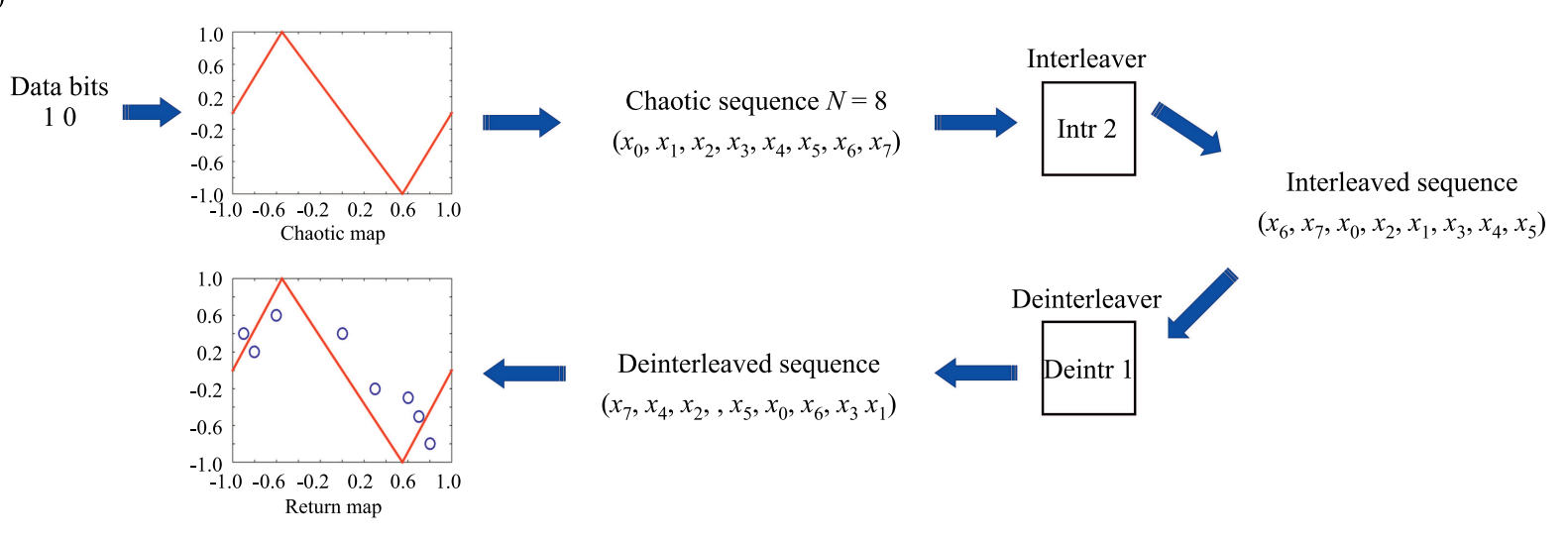

Fig. 2. M-ary modulation scheme: (a) correct interleaver pair and deinterleaver and (b) incorrect interleaver and deinterleaver pair.

\section{System Structure of Improved M-Ary Modulation}

Figure 1 shows a block diagram of the proposed improved $\mathrm{M}$-ary modulation scheme. The scheme uses $M$ interleavers at the transmitter and $M$ deinterleavers at the receiver. The number of interleaver/deinterleaver pairs equals $2^{b}$, where $b$ is the number of data bits. In this figure, two bits are transmitted, hence four pairs are used for data bits 00,01 , 10 and 11. These pairs are the core of separation and construction of the chaotic dynamics, so the performance of the system depends on their design. The decoding, performed by the deinterleaver and analysis blocks at the demodulator, requires that the separation patterns of the inter- 
leavers at the transmitter be aligned with the constraint conditions.

In this work, a random approach is used to allocate the numbers to separation patterns of each interleaver/deinterleaver pair. It is worth remembering that no separation pattern matched other patterns. Additionally, it is important that patterns do not overlap each other. For instance, the separated sequences when $N=8$ and $b=2$ are as follows:

- the original sequence: $x_{0}, x_{1}, x_{2}, x_{3}, x_{4}, x_{5}, x_{6}, x_{7}$,

- the interleaver (intr 0): $x_{1}, x_{7}, x_{2}, x_{0}, x_{4}, x_{3}, x_{5}, x_{6}$ for input data 00 ,

- the interleaver (intr 1): $x_{4}, x_{0}, x_{4}, x_{2}, x_{7}, x_{6}, x_{1}, x_{3}$ for input data 01 ,

- the interleaver (intr 2): $x_{6}, x_{7}, x_{0}, x_{2}, x_{1}, x_{3}, x_{4}, x_{5}$ for input data 10 ,

- the interleaver (intr 3): $x_{3}, x_{2}, x_{0}, x_{7}, x_{4}, x_{5}, x_{1}, x_{6}$ for input data 11.

\subsection{The Interleaver and Deinterleaver Structures}

In this section, more details about the operation of the interleaver and deinterleaver algorithm will be given. Figure 2 illustrates reconstruction and separation of the chaotic sequence - processes that are performed by applying the interleaver (intr) and deinterleaver (deint), respectively. In this figure, two cases are examined: correct and incorrect interleaver/deinterleaver pairs.

A zigzag chaotic map whose trajectory is shown in Fig. 3 is used for generating the chaotic sequence. When the transmitted data bits equal 10, the interleaver (intr 2) is used for separating the chaotic sequence at the transmitter, and only the corresponding deinterleaver (deint 2) can reconstruct the original sequence.

Figure 2 a shows a correct pair, where the original chaotic sequence is matched with the deinterleaved sequence. Therefore, the entire $x_{i}$ sequence falls in the trajectory of

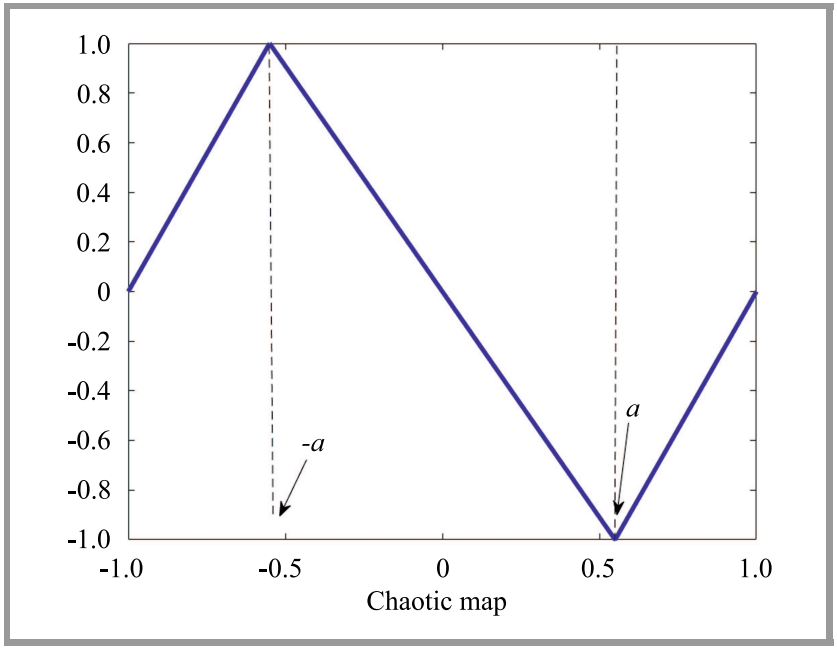

Fig. 3. Zigzag chaotic map. the zigzag map. Conversely, an incorrect pair shown in Fig. $2 b$ tends to point to a sequence that does not fall in the trajectory of the chaotic map (deint 1 is used instead of deint 2). Hence, based on chaotic dynamics, it is possible to reconstruct the sequence depending on whether it may be differentiated or not.

These characteristics, i.e. the loss of chaotic dynamics features, are noted for non-coherent communications based on chaos. The characteristics provide the receiver with additional information to decode the data correctly.

\subsection{The Transmitter Design}

The transmitter consists of $M$ interleavers and a chaotic signal generator, as indicated in Fig. 4. The chaotic sequence is generated using a chaotic zigzag map. The zigzag map is described by Eq. (1):

$$
x_{n+1}=\left\{\begin{array}{ll}
\frac{1}{1-a}\left(x_{n}+1\right) & -1 \leq x_{n} \leq-a \\
-\frac{1}{a} x_{n} & -a<x_{n} \leq a \\
\frac{1}{1-a}\left(x_{n}-1\right) & a<x_{n} \leq 1
\end{array},\right.
$$

where $n=0,1,2,3, \ldots, N-1 . \quad N$ is the length of the chaotic sequence and $a$ indicates the value of $x_{n}$ at which the minimum value of zigzag map trajectory occurs (due symmetry to the symmetry of the zigzag map, $-a$ would correspond to the maximum). In this case, an arbitrary interleaver (intr $m$ ) is selected, based on input data $m$. Subsequently, the chaotic sequence is linked to the selected (intr $m$ ), where the separation of the ordered samples takes place, following the separation patterns of (intr $m$ ) applied in separating the chaotic dynamics forming part of the sequence.

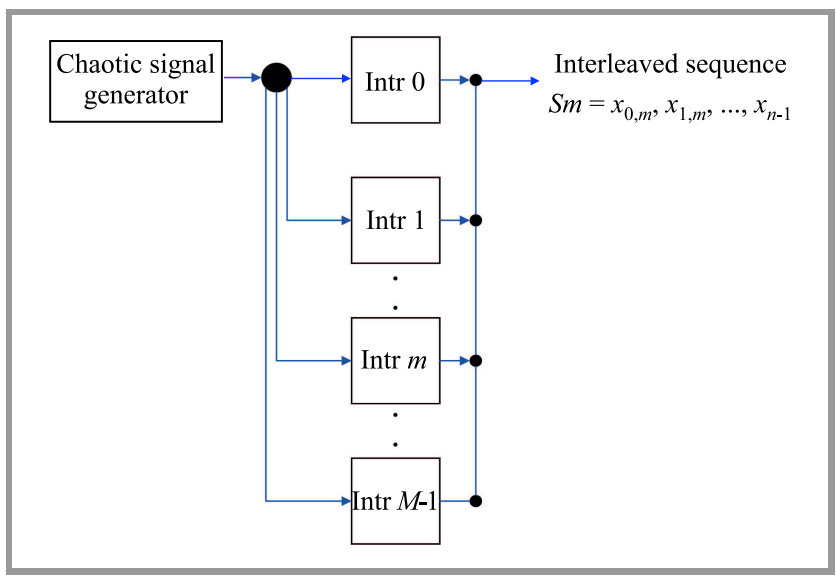

Fig. 4. Modulation system based on chaotic dynamics.

The chaotic sequence originally generated by the transmitter is given in Eq. (2) while the sequence after interleaving is given in Eq. (3):

$$
\begin{gathered}
S=x_{0}, x_{1}, \ldots, x_{i}, \ldots, x_{N-1}, \\
S=x_{0, m}, x_{1, m}, \ldots, x_{i, m}, \ldots, x_{N-1, m},
\end{gathered}
$$




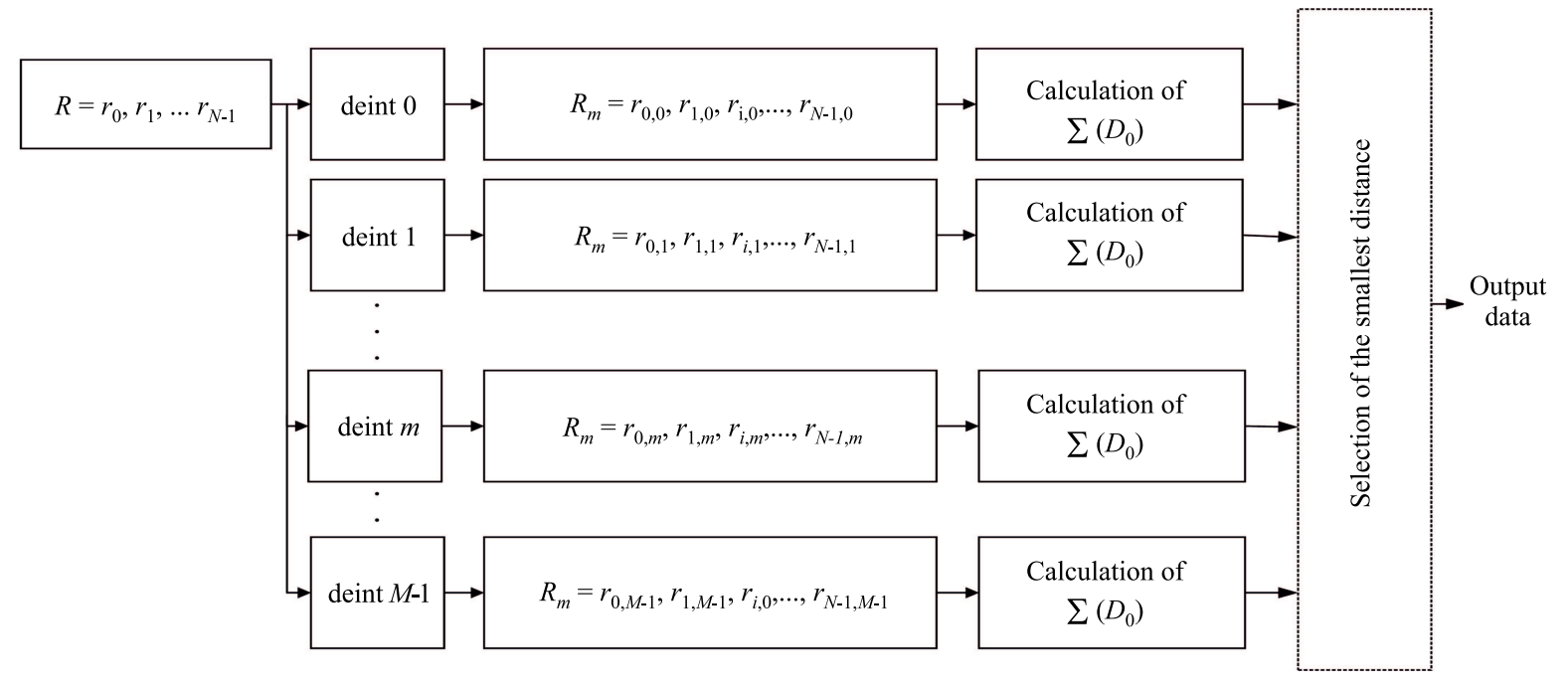

Fig. 5. Demodulation system based on the chaotic dynamics.

where, $x_{0}$ is the randomly selected initial value and $x_{i, m}$ is the $i$-th sample interleaved by (intr $m$ ). In the transmission channel, the signal would be distorted and corrupted by noise. In the current study, it is assumed that noise $n(t)$ in the channel is additive white with Gaussian probability distribution. So, the received sequence is expressed as $R=r_{0}, r_{1}, \ldots, r_{N-1}=S_{m}+n(t)$.

\subsection{Detection Algorithm Receiver}

The receiver encompasses the analysis unit of the chaotic dynamics $M$ deinterleavers, as shown in Fig. 5. Considering that the receiver is non-coherent, it helps memorize the chaotic zigzag map applied at the transmitter. So, the receiver is not aware of the initial value of the chaotic sequence. In this sense, the receiver is incapable of re reproducing the imitations of the base signals. Therefore, the receiver feeds the sequence to all deinterleaver elements a step that is is followed by the separation of the sequence orders using a deinterleaver (deint $m$ ). Equation (4) represents a deinterleaved sequence $R_{m}$ :

$$
R_{m}=r_{0, m}, r_{1, m}, \ldots, r_{i, m}, \ldots r_{N-1, m}
$$

where $r_{i, m}$ represents the sample of the sequence deinterleaved by (deint $m$ ). Every element of the deinterleaved sequence ${ }_{R} m$ becomes a part of the second unit, where the chaotic dynamics pave the way for the analysis of each sequence. This work applies a suboptimal detection algorithm to perform the analysis of chaotic dynamics. The algorithm is based on calculating the shortest distance between $N d$-successive signal samples which are generated by the zigzag map $(N d: 2,3, \ldots)$, and outputs the sum of the distances, achieving the minimum shortest distance within the set of $l$, between $i=0$ to $N-N d$.

$R_{i, m}^{\prime}$ is the $N d$-successive signal. We start with $i$ of the sequence deinterleaved by (deint $m$ ), where $l$ represents the straight lines in the $N d$-dimensional space. The parameters are given as:

$$
\begin{gathered}
R_{i, m}^{\prime}=r_{i, m}, r_{i+1, m}, \ldots, r_{i+N d-1, m} \\
l=3 \cdot 2^{N d-2}
\end{gathered}
$$

The calculation of the shortest distance requires finding the closest point $P_{l}$ between $R_{i, m}^{\prime}$ and the $l$-th line using the scalar product derived from the vector. Figure 6 shows the defined edges of the $l$-th line indicated by $P_{l}^{\prime}$ and $P_{l}^{\prime \prime}$, where $P_{l}$ is the closest point and it is calculated using:

$$
P_{l}=\left(p_{0}^{(l)}, p_{1}^{(l)}, \ldots, p_{N_{d}-1}^{(l)}\right)=\left(U_{l} \cdot V_{l}\right) U_{l}+P_{l}^{\prime}
$$

where unit vectors $U_{l}$ and $V_{l}$ are:

$$
\begin{gathered}
U_{l}=\frac{P_{l}^{\prime \prime}-P_{l}^{\prime}}{\left\|P_{l}^{\prime \prime}-P_{l}^{\prime}\right\|}, \\
V_{l}=R_{i, m}^{\prime}-P_{l}^{\prime} .
\end{gathered}
$$

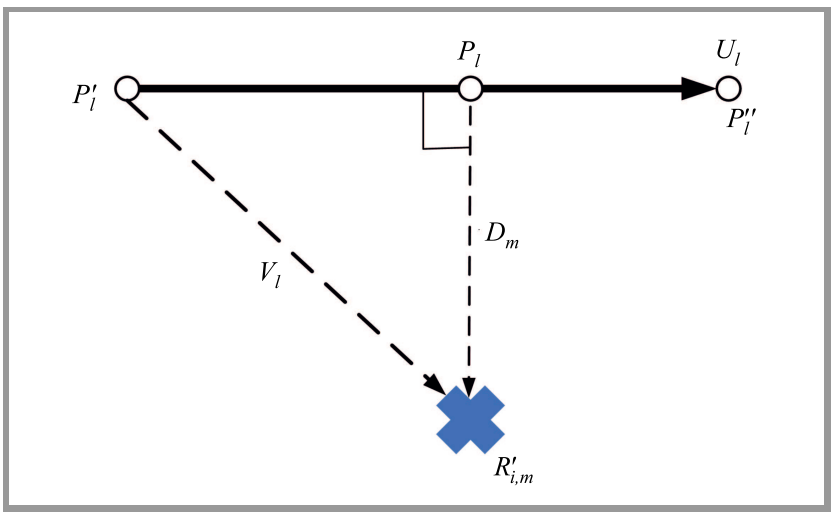

Fig. 6. Calculating the shortest distance. 
The distance is displayed as $\sum D_{m}$, and is calculated using the sequence deinterleaved by (deint $m$ ) as:

$$
\sum D_{m}=\sum_{i=0}^{N-N_{d}} \min || P_{l}-R_{i, m}^{\prime} \|
$$

Subsequently, the receiver selects the lowest $\sum D_{m}$ from the captured $\sum D_{m}$ values and data outputs. Considering the above description, the separating pattern of each interleaver-deinterleaver pairs ideally assumes different values. In this case, a single (correct) deinterleaver is capable of reconstructing the original sequences, created at the transmitter. Hence, the receiver outputs the symbol that corresponds to (deint $m$ ), for reconstructing $R_{m}$.

\section{Simulation Results}

Performance of the improved M-ary system is evaluated based on Matlab simulations. The simulation parameters used are as follows: zigzag parameter $a$ equals 0.55 and the initial value of the sequence is chosen randomly at the transmitter for each data transmitting point. In the simulations, these parameters are assumed to be $b=2,3$ and 4 , $M=4,8$ and 16 , and $N=32$. Therefore, in calculating the shortest distance, 4 -dimensional space $(N d=4)$ is considered. We repeated the simulation 104 times, evaluating performance in terms of the bit error rate (BER).

Figures 7a-c show the BER performance of the improved $\mathrm{M}$-ary modulation versus the traditional M-ary modulation versus the traditional $\mathrm{M}$-ary modulation based on chaotic dynamics for $M=4,8$ and 16, respectively. Based on these figures, we can see that at $\mathrm{BER}=10^{-3}$ the gains over the traditional method, obtained when using the improved method, at $M=4,8$ and 16 , are $1.6,1.7$, and $1.4 \mathrm{~dB}$, respectively. Furthermore, the gain obtained at BER $=10^{-1}$ is equals $3,2.8$ and $3.2 \mathrm{~dB}$, respectively. This gain is referred to as the increase in spreading factor of the chaotic signal. In addition, the zigzag map is a truly random variable and the chaotic signal is alternating between positive and negative values.

Figure 8 shows the BERs vs. Eb/N0 for improved M-ary at $M=4,8$ and 16. From this figure, we noticed that the improvement in BER performance increases as $M$ increases. For example, at $\mathrm{BER}=10^{-3}$, we recorded that the gain in $\mathrm{Eb} / \mathrm{NO}$ for $M=16$ over $M=4$ equals $2 \mathrm{~dB}$, and for $M=16$ over $M=8$ equals $0.6 \mathrm{~dB}$.

The increased computational complexity in terms of the number of multiplications required as $M$ increases constitutes one major challenge associated with the chaotic coding scheme. Based on the $6 N \times M \times 2^{N d-2}$ relation, there will be an increase in the number of multiplication operations. The relation could be explained by referring to Eqs. (7) and (10). Consistently with the two equations, the quantity of multiplication operations within all equations is equivalent to $N$. Therefore, the number of multiplications

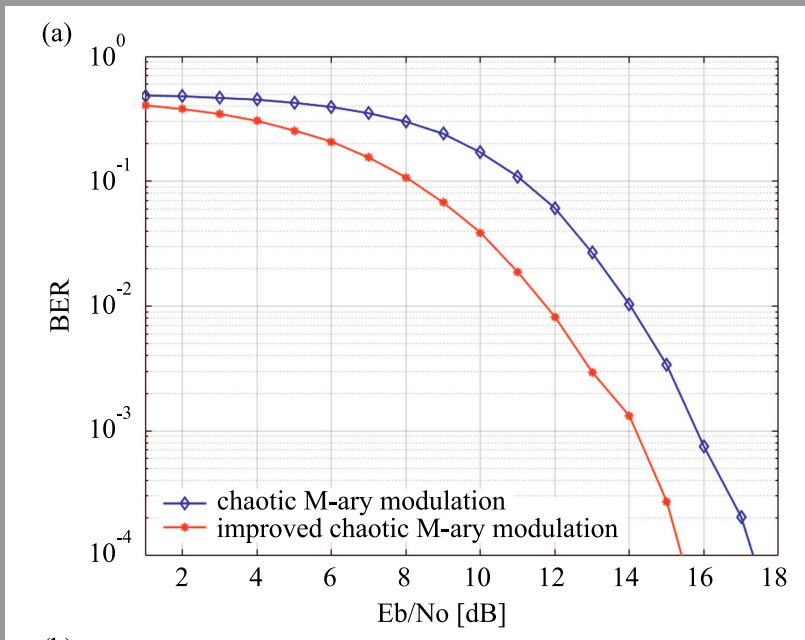

(b)

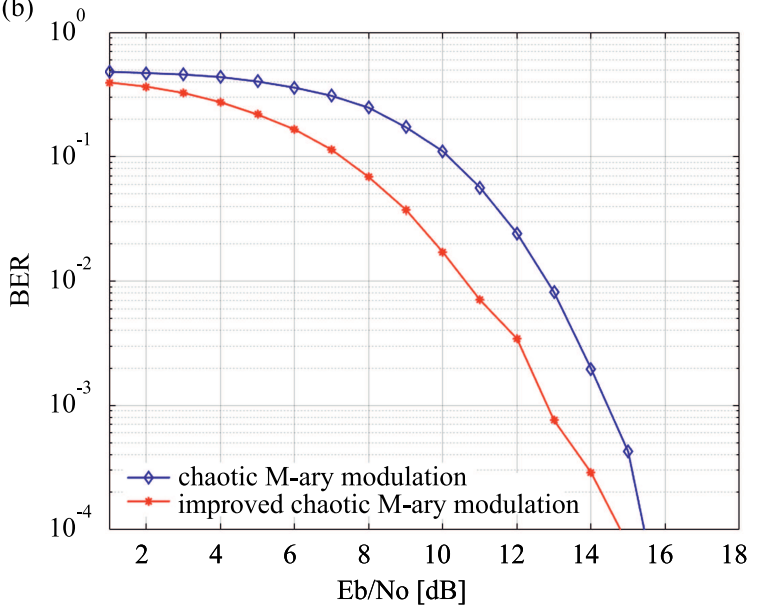

(c)

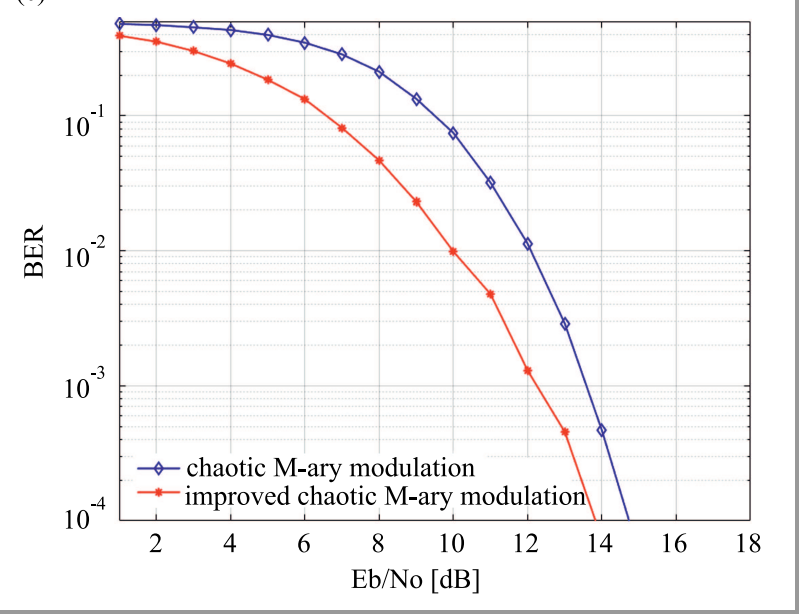

Fig. 7. BER performance of improved and traditional M-ary modulation: (a) $M=4$, (b) $M=8$, and (c) $M=16$.

within the two equations is $2 N$ and the operations would be replicated for every straight line. Because the number of straight lines is equivalent to $3 \times 2^{N d-2}$, the number of multiplication operations required would be $6 N \times M \times 2^{N d-2}$. The plot for the foregoing relation against $\mathrm{N}$ with $\mathrm{M}$ serving as the parameter is illustrated in Fig. 9. According to the figure, and consistently with the verified relation, it could be inferred that the number of multiplications re- 


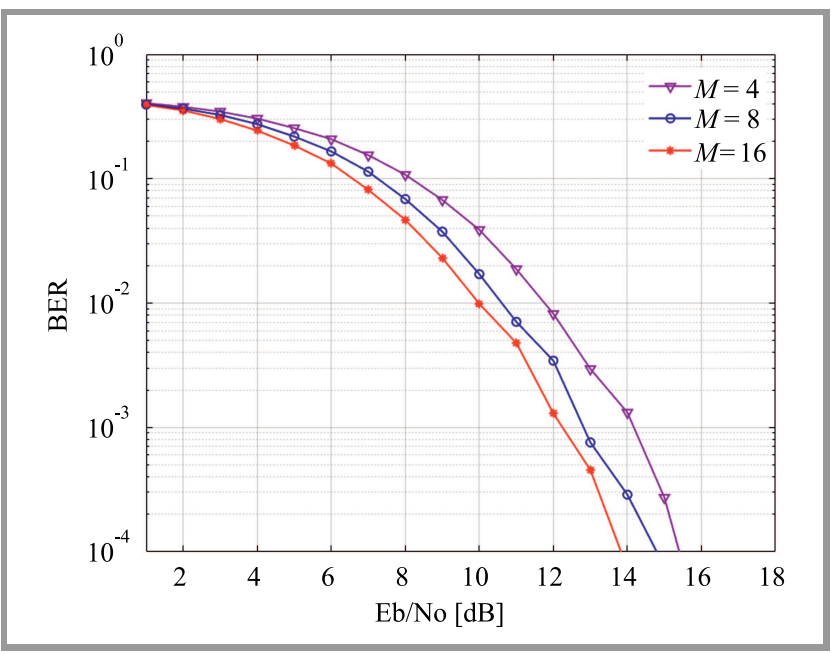

Fig. 8. BER performance for improved versus the traditional M-ary modulation: (a) $M=4$, (b) $M=8$, and (c) $M=16$.

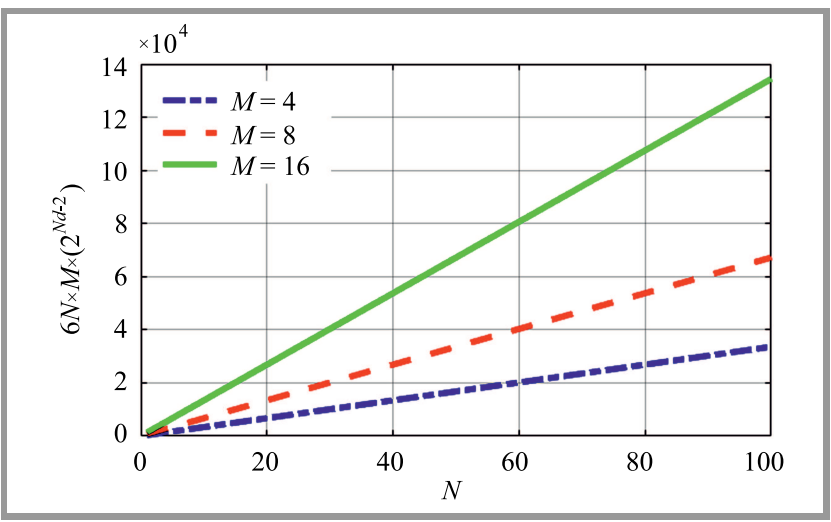

Fig. 9. Computational complexity of chaotic coding scheme with $M$ as parameter.

quired increases along with the increase in $M$. Notably, all multiplications could be considered real.

\section{Conclusions}

This paper has proposed an improved M-ary modulation scheme based on a zigzag map, through adoption of a noncoherent communication system based on chaos. The successive sequence of the chaotic signal is aligned to the chaotic dynamics in order to address the problem of reconstruction and separation of the chaotic dynamics of the sequence in question. Chaotic dynamics are relied upon as supplementary information in the correct recovery of the data sent. The improvement introduced by the proposed scheme becomes even more prominent as the number of interleaver/deinterleaver pairs used is increased.

\section{References}

[1] M. Hasler and T. Schimming, "Chaos communication over noisy channels", Int. J. of Bifurcation and Chaos, vol. 10, no. 4, pp. 719-736, 2000 (doi: 10.1142/S0218127400000505).
[2] H. N. Abdullah, T. R. Saeed, and A. H. Sahar, "Suboptimal detection of modified logistic map based chaos shift keying modulation", U. P. B. Scient. Bull. Series C, vol. 80, no. 3, pp. 85-94, 2018 [Online]. Available: https://www.scientificbulletin.upb.ro/ rev_docs_arhiva/rez389_313681.pdf

[3] F. J. Escribano, L. López, and M. A. F. Sanjuán, "Improving the performance of chaos based modulations via serial concatenation", IEEE Trans. on Circ. and Syst. I, vol. 57, no. 2, pp. 448-459, 2010 (doi: 10.1109/TCSI.2009.2019408).

[4] H. N. Abdullah and S. K. Mahmood, "Performance evaluation of non-redundant error correcting scheme using logistic chaotic map", J. of Wirel. Pers. Commun., vol. 86, no. 3, pp. 1169-1181, 2016 (doi: 10.1007/s11277-015-2981-2).

[5] G. Kaddoum, "Wireless chaos-based communication systems: A comprehensive survey", IEEE Access, vol. 4, pp. 2621-2648, 2016 (doi: 10.1109/ACCESS.2016.2572730).

[6] F. C. M. Lau and C. K. Tse, "On optimal detection of noncoherent chaos-shift-keying signals in a noisy environment", Int. J. of Bifurcation and Chaos, vol. 13, no. 6, pp. 1587-1597, 2003 (doi: 10.1142/S0218127403007448).

[7] H. N. Abdullah and A. Valenzuela, "Efficient chaotic communication system for wireless sensing applications", in Proc. of the 9th IEEE Int. Multi-Conf. on Syst., Sig. and Dev. SSD'12, Chemnitz, Germany, 2012, pp. 914-918 (doi: 10.1109/SSD.2012.6198019).

[8] Z. Liu, J. Zhang, and H. Liu, "Design of the differential chaos shift keying communication system based on DSP builder", Comp. Modell. \& New Technol., vol. 18, no. 12C, pp. 138-143, 2014 [Online]. Available: http://cmnt.lv/upload-files/ns_39crt_022.pdf

[9] W. Hu et al., "Non-coherent capacity of M-ary DCSK modulation system over multipath rayleigh fading channels", IEEE Access, vol. 5, pp. 956-966, 2017 (doi: 10.1109/ACCESS.2016.2623798).

[10] F. S. Hasan, "Design and analysis of an orthogonal chaotic vectors based differential chaos shift keying communication system", $A l$ Nahrain J. for Engin. Sci. (NJES), vol. 20, no. 4, pp. 952-958, 2017 [Online]. Available: https://www.nahje.com/index.php/main/ article/download/320/260

[11] G. Cai et al., "A square-constellation-based M-ary DCSK communication system", IEEE Access, vol. 4, pp. 6295-6303, 2016 (doi: 10.1109/ACCESS.2016.2612224).

[12] S. Arai, Y. Nishio, and T. Yamazato, "M-ary modulation scheme based on separation of deterministic chaotic dynamics for noncoherent chaos-based communications", Nonlin. Theory and Its Appl. IEICE, vol. 5, no. 2, pp. 210-221, 2014 (doi: 10.1587/nolta.5.210).

[13] H. Nejati, A. Beirami, and W. H. Ali, "Discrete-time chaotic-map truly random number generators: design, implementation, and variability analysis of the zigzag map", Analog Integr. Circ. Sig. Process, vol. 73, no. 1, pp. 363-374, 2012 (doi: 10.1007/s10470-012-9893-9).

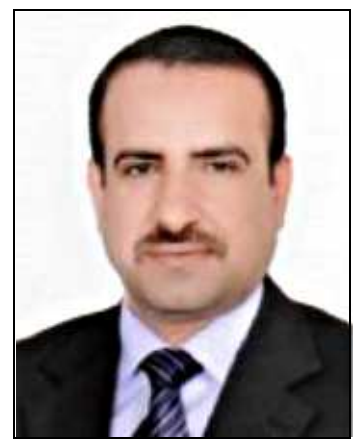

Hikmat N. Abdullah obtained his B.Sc. in Electrical Engineering in 1995, M.Sc. in Communication Engineering in 1998 from the University of AlMustansiryah, Iraq, and Ph.D. in Communication Engineering in 2004 from the University of Technology, Iraq. From 1998 to 2015, he worked as an associate professor at the Electrical Engineering Department of Al-Mustansiryah University, Iraq. Since the beginning of 2015, Abdullah has been working as a full professor at the college of Information Engineering of the Al-Nahrain University, Iraq. In 2011-2013 he received a research award from the International Institute of Education (IIE/USA) at Bonn-Rhein-Sieg University of Applied Sciences, Germany. He has been a senior mem- 
ber of IEEE since 2014. His research focuses on wireless communication systems and chaotic communications.

(iD) https://orcid.org/0000-0002-1133-2057

E-mail: dr.h.abdullah@ieee.org

Al-Nahrain University

College of Information Engineering

Baghdad, Iraq

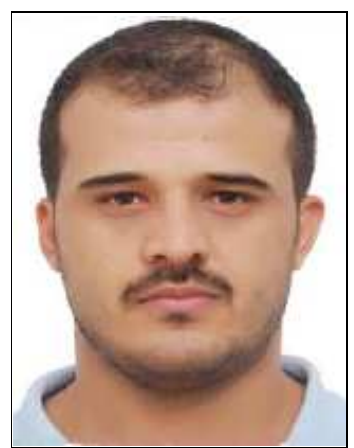

Asaad Hameed Sahar obtained his B.Sc. in Electronics Engineering in 2010, M.Sc. in Electronics Engineering in 2013, from the University of Technology, Baghdad, Iraq. In 2015 he joined a Ph.D. program in Electronic and Communication Engineering at the University of Technology.

E-mail: asaad.ha87@gmail.com

Department of Electrical Engineering

University of Technology

Baghdad, Iraq

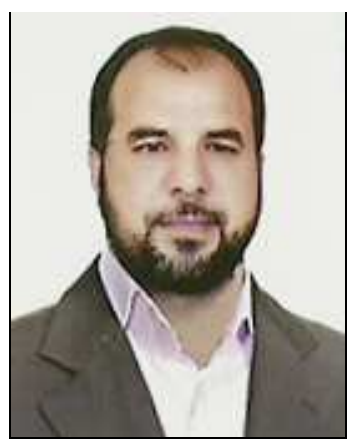

Thamir Rashed Saeed received his B.Sc. degree in 1987, and M.Sc. degree in 1994 both from the Military Engineering College in Baghdad. He also received the Ph.D. degree from the Al-Rashed College of Engineering and Science in Baghdad in 2003. From 1994 to 2003, he worked at the Military Engineering College in Baghdad, as a member of the teaching staff. He has been working, since, 2003, at the University of Technology in Baghdad, as a member of the teaching staff. Currently, he is an Assistant Professor of electrical engineering at the University of Technology. His major interests are in digital signal processing, digital circuit design for FPGA-based DSPs, radar, sensor networks and pattern recognition.

E-mail: thamir_rashed@yahoo.com

Department of Electrical Engineering

University of Technology

Baghdad, Iraq 